\title{
nature
}

photonics

\section{The lure of silicon}

\author{
Silicon has changed the world through microelectronic technology. Now optical researchers \\ are getting in on the silicon game too.
}

Look down at your feet. If you dig a little deeper you should stumble upon the second most abundant element on Earth: silicon. It makes up about $25 \%$ of the Earth's crust by weight ${ }^{1}$. But in the technology world it packs a much heavier punch. Silicon has revolutionized our lives by underpinning much of the microelectronic technology we see around us today.

When the Swedish chemist

Jöns Jakob Berzelius first isolated silicon as a chemical element in 1824 , he probably could not have imagined the impact his discovery would have. Today silicon is the workhorse of modern electronics and the backbone of our computer chips. The market for silicon devices is now worth about $\$ 200$ billion $^{2}$. The question is this: can silicon become a staple of photonic technologies too?

The will to siliconize photonics is strong. Over the next five to ten years, people will be clamouring for ever-increasing amounts of data. They will expect lightning-fast Internet access to deliver voice and video services in the blink of an eye, but the electrical lines that connect computer chips, boards and systems at present are not able to satisfy this appetite. Photons, which are faster and less lossy than electrons, offer a way forward. As Bahram Jalali of UCLA explains on page 193 of this issue ${ }^{3}$, optics integrated onto silicon could overcome the bandwidth limitations imposed by metallic interconnects between processors, heralding a light-driven information age. Companies such as Luxtera, IBM (who reported new optical buffers on silicon chips ${ }^{4}$ in our first issue in January) and Alcatel-Lucent's Bell Labs, not to mention governments, universities and funding bodies worldwide, are all throwing their pennies into the silicon wishing well.

But like many scientific goals, silicon photonics is not easy. Although silicon chips are cheap and can be mass-produced, they are nowhere near as light-friendly as more exotic,

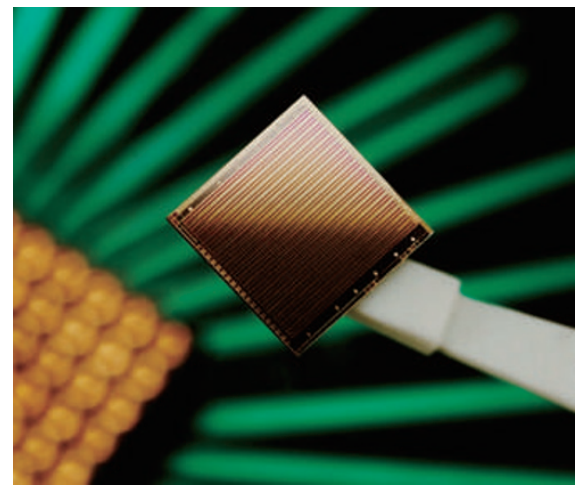

expensive semiconductors, such as indium phosphide and gallium arsenide. If silicon is to play ball, it must be able to support light generation, transport and detection - all in an efficient, miniature package. Intel, which controls more than $80 \%$ of the microprocessor market and is one of the biggest hitters in the silicon-chip game, is now immersed in the first of three phases of its silicon dream. According to Sean Koehl", "The first phase is to prove silicon's viability as an optical material." In this issue, Haisheng Rong and co-workers at Intel take us a step closer to a low-cost silicon light-source ${ }^{6}$. As the team explains in our back-page interview ${ }^{7}$, they have produced a silicon Raman laser that is more practical than ever before. Such lasers have the potential to revolutionize not only optical communications, but also impact medicine and sensing applications too.

In reality, optical chips may end up relying on both silicon and III-V-family semiconductor materials to get the best of both worlds. As expressed at a recent Optoelectronic Industry Development Association silicon-photonics forum, the way to break down the optical interconnect 'brick wall' is to intensively research a number of solutions, which could quite conceivably involve exploiting optoelectronic technology from the III-V community ${ }^{2}$. Late last year, a collaboration of scientists from Intel and the University of California, Santa Barbara, built the world's first electrically driven 'hybrid silicon laser' by harnessing the light-emitting properties of indium phosphide and the lightrouting abilities and low cost of silicon.

Ironically, as one area of optics moves towards silicon, another wishes it wasn't quite so dependent on this wonder material. According to the International Technology Roadmap for Semiconductors, within a few years the rapidly expanding solar-cell industry will overtake the chip sector in its use of silicon ${ }^{8}$. However, an acute shortage of silicon - in particular, the polycrystalline version used in photovoltaic devices threatens to curb growth of this alternativeenergy field. The European Commission is calling for $20 \%$ of European Union energy consumption to be met by renewable sources by 2020 . Energy-conscious governments, such as those in Germany and Japan, are heavily subsidizing solar technology. But polysilicon prices are now about five times what they were in 2004, and industry insiders say this could disrupt the drive for costeffective photovoltaics for the next decade.

Nevertheless, if the push for silicon photonics has taught us one thing, it is that where there's a will, there's a way. The desire for silicon-based optical technologies - be they within our computers or on our roofs - shows no sign of abating. Silicon has made its mark on the electronics industry, and now it is stamping its authority on the photonics world too.

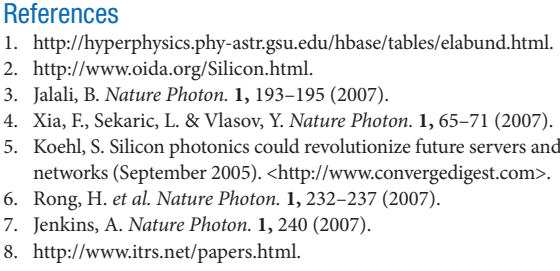

